\title{
Causal Knowledge and Reasoning in Decision Making
}

\author{
York Hagmayer ${ }^{* 1}$ and Cilia Witteman \\ *University of Goettingen, Goettingen, Germany \\ ${ }^{\S}$ Radboud University, Nijmegen, The Netherlands \\ ${ }^{1}$ Corresponding author: E-mail: york.hagmayer@bio.uni-goettingen.de
}

\section{Contents}

1. Introduction

2. Causal Knowledge and Reasoning in Decision Making 98

2.1 Decision Making Based on Expected Outcomes 99

$\begin{array}{ll}2.2 \text { Decision Making Based on Narratives } & 105\end{array}$

2.3 Decision Making Based on Cues 108

2.4 Do Causal Knowledge and Reasoning Improve Decision Making? 110

$\begin{array}{ll}2.5 \text { Summary } & 115\end{array}$

3. Integrating Causal Knowledge and Reasoning Into 116

Decision Making - the Causal-Explanation-Based Decision-Making Framework

3.1 Step 1: Decide on Causal Analysis

3.2 Step 2: Provide a Causal Explanation for the Problem 119

3.3 Step 3: Determine the Utility of Possible Interventions 121

3.4 Step 4: Decide on an Intervention 122

3.5 Step 5: Evaluate the Intervention and Causal Explanation 124

$\begin{array}{ll}3.6 \text { Summary } & 125\end{array}$

4. Conclusions 125

References 128

\section{Abstract}

Normative causal decision theories argue that people should use their causal knowledge in decision making. Based on these ideas, we argue that causal knowledge and reasoning may support and thereby potentially improve decision making based on expected outcomes, narratives, and even cues. We will summarize findings from empirical research, which indicate that decision makers use their causal knowledge and reasoning when making a choice. Focusing on decision making in mental health, we will also show that at least sometimes the outcomes resulting from a chosen action can be improved by taking causal knowledge into account. In the second part of the chapter, we propose a structured process describing when and how decision makers should use causal knowledge and reasoning in decision making: the Causal-explanation-based Decision Making framework. Mental health will again 
be our exemplary domain. We will end by arguing that our model may serve as a blueprint for a rational model for clinical decision making, which may also guide future research.

\section{INTRODUCTION}

Causal knowledge and reasoning are assumed to play a central role in human thought (Gopnik \& Schulz, 2007; Kahneman, 2011; Sloman, 2005; Waldmann, 1996; Waldmann, Cheng, Hagmayer, \& Blaisdell, 2008) and they are assumed to serve several different functions (Danks, 2014). Causal knowledge allows reasoners to provide an explanation for the occurrence of an event, e.g., the loss of a loved one may explain why a person experiences an episode of depression. Causal knowledge and reasoning also allow reasoners to predict future events and even the consequences of counterfactuals. For example, it allows a therapist to envision consequences of confronting a person suffering from posttraumatic stress disorder (PTSD) with stimuli associated with the trauma.

The second function of causal knowledge and reasoning is also epistemic. Causal knowledge enables causal learning (Danks, 2014; Tenenbaum, Kemp, Griffiths, \& Goodman, 2011). Computational analyses and simulation studies show that the data usually available to learners in everyday life would be too few to allow for reliable and valid inferences if learners had no previous causal knowledge, which constrains the number of possible hypotheses (Tenenbaum et al., 2011). Without knowledge about the type of stimuli that usually causes panic attacks it would be impossible for a clinician to determine, by looking at selfmonitoring protocols, which stimuli trigger the attacks of particular patient.

Causal knowledge and reasoning also serve pragmatic functions. They allow people to regulate their emotions in a way that enhances their well-being (cf. Weiner, 1985). For example, by attributing failure to conditions outside the person's control, the person avoids feeling guilty or ashamed and can continue to believe in high capability. Causal attribution also helps to regulate motivation. By attributing success to personal effort, the motivation to spend a lot of effort is increased.

Maybe the most important function of causal knowledge and reasoning, though, is to support decision making. When people make decisions, they usually do so to achieve certain desirable outcomes. Causal knowledge 
and reasoning can help decision makers predict the consequences that might result from the different courses of actions they could take. Thereby it supports decision makers in selecting the course of action that is most effective in generating a desired outcome. This function will be the focus of this chapter.

We pursue two goals. In the first part of the chapter we intend to show how causal knowledge and reasoning affect decision making based on expected outcomes, narratives, and even cues. We will outline how these decision-making strategies may profit from causal knowledge and inferences derived from this knowledge. In addition, we will present evidence from the literature on clinical decision making, which indicates that causal knowledge and reasoning may result in better outcomes for the patient.

In the second part of the chapter we show how causal knowledge and reasoning could be integrated into a unified framework of what we call Causal-explanation-based decision making (CDM). The framework describes when and how causal knowledge and reasoning should be used in a structured process to improve the choices made. We will point out findings, which show that decision makers are able to follow these recommendations.

In the end, we will discuss how this framework might be used as a blueprint for a rational model of causal reasoning in decision making. We will show what further steps need to be taken, both in developing the theory and in providing empirical support for its claims.

Throughout the chapter we will focus on treatment decision making in mental health. There are two main reasons for doing so. The first reason is that clinicians are supposed to consider causal knowledge when they decide on a treatment. Proponents of evidence-based practice in medicine (e.g., Sackett, Rosenberg, Muir Gray, Haynes, \& Richardson, 1996) and psychotherapy (e.g., APA Presidential Task Force on Evidence-Based Practice, 2006; Dozois et al., 2014) ask clinicians to take knowledge about the causal effects of different treatments into account when making a decision for a particular patient. More precisely, clinicians shall use the current best evidence from empirical research, the specific characteristic of their patient, his or her treatment preferences, and their personal experience as a clinician to make decisions about care. Clinicians shall consider evidence and experience to select the intervention(s) that maximizes the chance of benefit and minimize the risk of harm (cf. Dozois et al., 2014). 
The second reason to look at clinical decision making is that empirical research has shown that mental health clinicians (including psychologists and psychiatrists) have causal knowledge that they could use when deciding on a treatment. For example, Ahn, Proctor, and Flanagan (2009) investigated clinicians' assumptions about the causes of the disorders listed in the Diagnostic and Statistical Manual of Mental Disorders (DSM IV, APA, 2000). They found that clinicians ordered disorders on a single dimension ranging from disorders caused by biological factors over disorders caused by both biological and psychosocial factors to disorders caused only by psychosocial and environmental factors. DeKwaadsteniet and Hagmayer (2017) showed that clinicians hold rather elaborate causal theories about how biological, social, and cognitive factors contribute to the symptoms characterizing developmental disorders. Importantly, clinicians also had assumptions about how interventions target the factors and/or symptoms. Finally, Kim and Ahn (2002) found that clinicians have theories about how symptoms of a disorder are causally connected to each other.

These two reasons make clinical decision making a domain that is especially suitable for our endeavor. Clinicians have causal knowledge relevant to treatment choice, and they are required to use it, because doing so is expected to yield better outcomes. Therefore we would expect to find that causal knowledge and reasoning (1) affect clinical decision making and (2) lead to choices that result in better health.

\section{CAUSAL KNOWLEDGE AND REASONING IN DECISION MAKING}

Many different strategies for making a decision have been described in the literature (cf. Koehler \& Harvey, 2004). Some of these strategies are more likely to be affected by causal knowledge and reasoning (e.g., decision making based on expected outcomes). Other strategies seem to be independent of causal considerations (e.g., decision making based on social norms or current emotions). In this first part we will focus on decision-making strategies that may be affected by causal knowledge: decision making based on expected outcomes, narratives, and cues. We will outline how causal knowledge and/or inferences derived from this knowledge may support decision making and result in a choice that is more likely to yield a desired outcome. 


\subsection{Decision Making Based on Expected Outcomes}

Expected Utility Theory (Savage, 1954; Von Neumann \& Morgenstern, 1947) is still considered to be the gold standard of rational decision making. The theory distinguishes between actions $\left(\mathrm{A}_{\mathrm{i}}\right)$, outcomes $\left(\mathrm{O}_{\mathrm{ji}}\right)$, utilities of outcomes $\left(\mathrm{U}\left[\mathrm{O}_{\mathrm{ji}}\right]\right)$, and the situation $\left(\mathrm{S}_{\mathrm{j}}\right)$. The theory assumes that a utility can be assigned to every outcome, which captures how beneficial the outcome would be. It also assumes that every outcome can be assigned a probability $P\left(\mathrm{O}_{\mathrm{ji}}\right)$, which represents the uncertainty that the outcome will happen. Outcomes are usually uncertain even when the situation and action are known, because causal relations in the world tend to be probabilistic (i.e., $\left.P\left(\mathrm{O}_{\mathrm{ji}} \mid \mathrm{S}_{\mathrm{j}}, \mathrm{A}_{\mathrm{i}}\right)<1\right)$. According to the theory a rational decision maker has to calculate the expected utility $(\mathrm{EU})$ of every action $\mathrm{A}_{i}$ by multiplying the probability of each possible outcome with the utility of the respective outcome and summing up the products over all outcomes that could result from this action (i.e., $\left.\mathrm{EU}\left(\mathrm{A}_{\mathrm{i}}\right)=\sum_{\mathrm{ji}} P\left(\mathrm{O}_{\mathrm{ji}} \mid \mathrm{S}_{\mathrm{j}}, \mathrm{A}_{\mathrm{i}}\right) * P\left(\mathrm{~S}_{\mathrm{j}}\right) * \mathrm{U}\left(\mathrm{O}_{\mathrm{ji}}\right)\right)$. To be rational, the action with the highest expected utility should be chosen, that is, the action that maximizes expected utility should be preferred (principle of maximizing expected utility).

Apart from expected utility theory as a normative theory, many descriptive theories of decision making have built upon the idea that decisions are based on the expectance and value of outcomes of available options (e.g., Vroom, 1964). Prospect theory (Kahneman \& Tversky, 1979) is probably the most prominent example. Although it assumes that decision makers transform probabilities of outcomes in a nonlinear way and estimate the value of an outcome relative to a reference point, it still assumes that decision makers base their decision on the expectancies and values of outcomes.

Proponents of causal decision theory point out that decisions based on expected utility may be suboptimal when the causal relations underlying the probabilistic relation between action and outcome are not properly considered in choice (Joyce, 1999; Lewis, 2006; Maher, 1987; Nozick, 1993; Skyrms, 1982). Expected utility theory takes into account the probabilistic relation between potential actions, possible states of the world, and outcomes, $P\left(\mathrm{O}_{\mathrm{ji}} \mid \mathrm{S}_{\mathrm{j}}, \mathrm{A}_{\mathrm{i}}\right)$. But it ignores that the same probabilistic relation between an action and an outcome may result from different causal structures. An action and an outcome may be positively related (i.e., $\left.P\left(\mathrm{O}_{\mathrm{j} 1} \mid \mathrm{S}_{\mathrm{j}}, \mathrm{A}_{1}\right)>P\left(\mathrm{O}_{\mathrm{j} 1} \mid \mathrm{S}_{\mathrm{j}}, \sim \mathrm{A}_{1}\right)\right)$ because of several causal structures. First, 
the action may generate the outcome. For example, antidepressant medications probabilistically generate a better mood in patients suffering from major depression. Second, the action may not itself generate the outcome but enable another factor to generate the outcome. For example, many patients who suffer from PTSD also abuse drugs or other substances. Detoxification does not improve the symptoms of PTSD itself but provides the basis for other effective treatments such as cognitive behavior therapy. Third, the action may change the situation, which in turn affects the outcome. Family therapy for eating disorders, for example, does not address the dysfunctional eating, but it changes social interactions within the family that maintain the patient's disorder. Finally and most importantly, the action and the outcome may just be spuriously related. This is the case when the action and the outcome are affected by a common cause, but the action has no impact on the outcome. For example, debriefing is a popular intervention after potentially traumatizing events, and it seems to have a positive statistical relation with the reduction of symptoms of posttraumatic stress. But there is no evidence that debriefing has a positive influence on the symptoms (Wessely, Bisson, \& Rose, 2000). A spurious relation may result, if debriefing is given more often after severe events when the symptom load is high. Within the first 4 weeks after the incidence the symptom load goes down automatically, which implies a large reduction of symptoms when the symptom load was high after the event. After more minor incidences no debriefing is given. As the symptom load is lower than after major incidences, it goes down less. In consequence, there is a positive relation between debriefing and the reduction of symptoms, which is entirely spurious.

To calculate the expected utility of an action only probabilities that reflect causal relations but not spurious relations should be taken into account (Nozick, 1993), that is, causal expected utilities (CEU) should be calculated. In the first three cases causal expected utility should be calculated by $\operatorname{CEU}\left(\mathrm{A}_{\mathrm{i}}\right)=\sum_{\mathrm{ji}} P\left(\mathrm{O}_{\mathrm{ji}} \mid \mathrm{A}_{\mathrm{i}}, \mathrm{S}_{\mathrm{j}}\right) * P\left(\mathrm{~S}_{\mathrm{j}}\right) * \mathrm{U}\left(\mathrm{O}_{\mathrm{ji}}\right)$, because the action has a direct or indirect causal influence on the outcome. When the action and the outcome are spuriously related, though, the outcome and the action are independent of each other, formally $P\left(\mathrm{O}_{\mathrm{ji}} \mid \mathrm{A}_{\mathrm{i}}, \mathrm{S}_{\mathrm{j}}\right)=P\left(\mathrm{O}_{\mathrm{ji}} \mid \mathrm{S}_{\mathrm{j}}\right)$. Therefore causal expected utility has to be calculated as $\operatorname{CEU}\left(\mathrm{A}_{\mathrm{i}}\right)=\sum_{\mathrm{ji}} P\left(\mathrm{O}_{\mathrm{ji}} \mid \mathrm{S}_{\mathrm{j}}\right) * P\left(S_{\mathrm{j}}\right) * \mathrm{U}\left(\mathrm{O}_{\mathrm{ji}}\right)$. 
Sloman and Hagmayer (2006) point out that it is not only important to consider causal structures in decision making but also to distinguish between observing an action and actively choosing an action (see also Fernbach, Sloman, \& Hagmayer, 2014; Hagmayer \& Sloman, 2009). When there is a direct or indirect causal relation between an action and an outcome, then an observed probabilistic relation is indicative of the underlying causal relation, and the observed probabilities can be used to estimate the causal expected utility of the respective action. When there is a spurious relation between an action and an outcome, observing the action is predictive of the outcome, but choosing the action does not affect the likelihood of the outcome. Hence, it would be wrong to use observed probabilities to estimate causal expected utility. Consider the example of debriefing again. If you observe someone getting debriefing after a traumatizing event, the person is likely to experience a large reduction of symptoms within the next four weeks. If you observe a person not to get debriefing after an event, the person is less likely to experience a large reduction in symptoms. Hence $P\left(\mathrm{O}_{\mathrm{ji}}=\right.$ large reduction $\mid \mathrm{A}=$ debriefing, $\left.\mathrm{S}_{\mathrm{j}}\right)>P\left(\mathrm{O}_{\mathrm{ji}}=\right.$ large reduction $\mid \mathrm{A}=$ no debriefing, $\left.\mathrm{S}_{\mathrm{j}}\right)$ when the action is observed. But this relation is not relevant for deciding whether to give debriefing. For decision making the consequences of actively choosing an action are relevant. When the relation between an action and an outcome is spurious, then taking this action will not affect the outcome. Thus irrespective of whether the action is taken, the probability of the outcome remains the same. For debriefing that means that the probability of achieving a large reduction of symptoms will be same regardless of whether debriefing will be given or not, formally $P\left(\mathrm{O}_{\mathrm{ji}}=\right.$ large reduction $\mid \mathrm{A}=$ debriefing, $\left.\mathrm{S}_{\mathrm{j}}\right)=P\left(\mathrm{O}_{\mathrm{ji}}=\right.$ large reduction $\mid \mathrm{A}=$ no debriefing, $\left.\mathrm{S}_{\mathrm{j}}\right)$ when $A_{i}$ is actively chosen. This is because by virtue of choosing the action, the action becomes independent of the common cause. The action now depends on the decision makers' choice and not on the common cause.

Based on these theoretical ideas and causal model theories of learning and reasoning (Waldmann, 1996; Sloman, 2005), Sloman and Hagmayer (2006) proposed the causal model theory of choice as a descriptive model of decision making. A causal model is a (mental) representation of a causal structure in the world. Causal models represent the direction and strength of causal relations between the considered entities (usually events or states). They allow a reasoner to predict the consequences of potential actions (Fernbach, Darlow, \& Sloman, 2010; Sloman \& Lagnado, 2005; Waldmann \& Hagmayer, 2005). According to the causal model theory 
of choice, decision makers go through three phases. First, the decision maker represents the decision problem as a causal model. This model captures the relevant outcome variables, their potential causes, and the directions and strengths of the causal relations. For example, to decide on a treatment for depression a causal model of the patient's problems will be constructed. Some potential causes may be observed (e.g., loss of a loved one) others may be inferred from the observed symptoms using the causal model (e.g., lack of coping skills). In the second phase, the available actions are added to the model. According to the theory, these actions are considered interventions on variables within the causal model. Interventions may target outcomes directly (e.g., mood-enhancing drugs reducing negative emotions) or causes of outcomes (e.g., training for coping skills). The model is then used to infer the consequences that would result from the different actions by running mental simulations. These simulations take into account uncertainties, including uncertainties about the causal relations. For example, in the case of depression treatment, the consequences of various treatments and treatment combinations are envisioned taking into account that a treatment may not work for a particular patient. In the final phase, a decision is made based on the results of the simulations. If an option has no impact on the desired outcome (i.e., the action and the outcome are just spuriously related) or its costs outweigh its benefits, it is discarded straight away. If there is only one option that increases the likelihood of the desired outcome, the option is chosen. If several options make the desired outcome more likely, the theory assumes that decision makers adapt their decision-making strategy to the given circumstance. If there is time pressure, decision makers may prefer to take the first option that increases the likelihood of desired consequences to a sufficient degree. If there is enough time and the consequences of the decision are important, the decision maker may prefer to search for the option that maximizes causal expected utility. In the case of depression, one clinician may immediately decide on drug treatment because it directly improves the patient's mood, while another clinician may prefer psychotherapy to target deficits in coping, because good coping skills will improve the patient's condition and protect him or her in the future.

Do causal knowledge and reasoning affect decision making based on expected outcomes? One prediction that can be derived from causal decision theories is that decision makers will consider the causal consequences that would result from the different available options for action. Huber et al. investigated which information people search for before making a choice 
between different options to achieve a given set of goals (Huber, Huber, \& Bär, 2011; Huber, Wider, \& Huber, 1997). They found that decision makers wanted to know about the causal consequences of the different options, first positive ones and then negative ones. Decision makers were also interested in exploring additional actions that might counteract possible negative consequences arising from the different options ("risk-diffusing operators," Huber et al., 1997).

Other experiments studied whether decision makers analyze the causal structure that relates actions and outcomes. Hagmayer and Sloman (2009, Experiment 2) investigated whether decision makers activate knowledge about the causal relation that underlies a statistical relation between an action and an outcome when asked to recommend a course of action. For example, participants were told that people who watch movies in their original language, speak better English than people who do not. Then participants were asked whether they would recommend taking the action to a friend, who desires to achieve the outcome. After participants made their recommendation, their assumptions about the underlying causal structure were assessed (a common cause influencing the action and the outcome or a direct causal relation). It turned out that participants who believed in a common cause did not recommend taking the action, while people who believed in a direct causal relation did. Hagmayer and Meder (2013) showed that decision makers spontaneously start to learn about the causal structure connecting actions to outcomes when making the same choice repeatedly. Participants did learn about causal structure, although these inferences were not necessary to pick the best course of action. The acquired assumptions about causal structure strongly affected later decisions when new options became available. Conflicting evidence, however, comes from studies on the control of dynamic systems, which indicate that participants only sometimes consider the underlying causal structure (Hagmayer, Meder, Osman, Mangold, \& Lagnado, 2010; Osman, 2010) or may fail to derive the right predictions from the causal structure when it contains feedback loops (Sterman, 2000).

There are several studies indicating that people base their decision on CEU of the available actions (Hagmayer \& Meder, 2013; Hagmayer \& Sloman, 2009; Robinson, Sloman, Hagmayer, \& Hertzog, 2010). In these studies the probabilistic relations between actions and outcomes were held constant. Nevertheless participants made differential recommendations and choices, depending on their assumptions about the underlying causal structure. In consequence, participants preferred the action that maximized 
causal expected utility. Indirect evidence for the hypothesis that people decide based on CEU comes from studies on clinical decision making. Flores et al. showed that clinicians prefer interventions that target the first event within a causal chain (Flores, Cobos, Lopez, \& Godoy, 2014; Flores, Cobos, López, Godoy, \& González-Martín, 2014; see also Yopchick \& Kim, 2009). Additional findings pointed out that this preference was due to clinicians' assumption that by addressing the first cause, all variables within the chain would be changed in desirable way. Given this assumption, an intervention on the first cause would result in the highest causal expected utility. Related findings were presented by DeKwaadsteniet, Hagmayer, Krol, and Witteman (2010), who showed that clinicians rate treatments as more effective if they change many causal factors that contribute to a client's problems.

One study investigated whether decision makers are sensitive to the distinction between observing an action and choosing an action when inferring the probability of consequences (Hagmayer \& Sloman, 2009; Experiment 3). In this experiment, participants were asked to predict the probability of an outcome given than an action was chosen, enforced by another person or machine, or observed. In all conditions participants were presented with the same probabilistic relation between an action and an outcome. The relation was either explained as being due to a direct causal relation or as being due to a common cause. It turned out that participants made the same predictions for deliberate choices and externally enforced actions. In these conditions participants expected to get the outcome if they assumed a direct causal relation, but not when they assumed a common cause. By contrast, when the action was observed, participants expected the outcome regardless of causal structure. In the common cause condition, this finding indicates that participants expected the common cause to be present when the action was observed. Therefore they expected the outcome.

Several studies on causal reasoning and learning investigated whether people are able to derive predictions about the consequences of interventions from observations and knowledge about the underlying causal structure (Meder, Hagmayer, \& Waldmann, 2008, 2009; Sloman \& Lagnado, 2005; Waldmann \& Hagmayer, 2005). Participants in these studies were asked to infer the likelihood of consequences of interventions they had never taken or observed before. The findings showed that participants could make these inferences. This result strengthens the hypothesis that 
decision makers can decide based on CEU if they have the required causal knowledge.

However, some studies indicate that people may sometimes prefer actions that reduce CEU. Studies on self-deception and self-handicapping show that ordinary people may sometimes hurt themselves although no positive consequences will result from the self-inflicted harm (Bodner \& Prelec, 2003; Fernbach, Sloman, \& Hagmayer, 2014; Mijovic-Prelec \& Prelec, 2010; Quattrone \& Tversky, 1984; Sloman, Fernbach, \& Hagmayer, 2010). For example, participants in Quattrone and Tversky's (1984) study endured the pain of cold water longer, if they believed that it indicated a good type of heart than when they believed it indicated a weak heart. They did so although the endured pain had no causal impact on the type of heart they had. Thus, they violated the principle of maximizing causal expected utility.

In sum, the empirical findings show that causal knowledge and inferences based on this knowledge affect decision making. Moreover, they support many predictions that can be derived from causal decision theories: decision makers analyze the causal structure underlying relations among actions and outcomes, they infer the likelihood of outcomes that would result from actively choosing an action, and they often prefer actions that maximize causal expected outcomes.

\subsection{Decision Making Based on Narratives}

The primary function of a narrative is to make sense of an observed sequence of events and to explain how a certain situation came about (Pennington \& Hastie, 1992). A narrative represents the sequence of events as it probably happened. Although their primary function is not decision making, narratives can provide a basis for decision making. They can do so it two ways. First, a narrative identifies the factors that causally contributed to a certain event (e.g., the onset of a dissociative disorder) or situation (e.g., an episode of major depression). Therefore a narrative allows the decision maker to target the causes of a current situation with their actions. For example, if the narrative indicates that a patient's depressive symptoms are caused by a lack of activities leading to positive reinforcement, the treatment may focus on identifying and practicing such activities.

Second, a certain type of narrative may be linked with a certain type of decision, as is illustrated in medical decision making. Diseases very often follow a certain course, that is, symptoms occur in a specific order and with specific delays in between. Hence, the development of symptoms 
over time indicates a particular disease. In mental health, there are no unique courses for each disorder, but there are typical patterns. For example, patients with agoraphobia often start to anticipate being anxious in public places and then begin to avoid going there, which results in a negative reinforcement of avoidance (vicious cycle of anxiety, Barlow, 2002). There is some indication that clinicians represent the typical causal sequences of events as illness scripts (Charlin, Boshuizen, Custers, \& Feltovich, 2007; Feltovich \& Barrows, 1984), which represent not only how symptoms change over time, but also the underlying causal mechanisms. In addition, illness scripts include typical treatments and their expected consequences. When a new patient presents with her or his problems, the information on the development of the problems over time allows the clinician to construct a narrative. This narrative can then be matched to illness scripts, which imply certain diagnoses and treatments.

The most prominent descriptive model of narrative-based decision making is the story model (Pennington \& Hastie, 1992), which has been developed to account for juror decision making in legal cases. It can also be used to model decision making in other areas. The theory assumes that decision makers spontaneously start to construct a narrative when presented with information on a case (see Pennington \& Hastie, 1986). The decision maker tries to reconstruct the actual temporal and causal order of events. Events include actions, physical events, and mental states, which are connected through physical and/or mental causation. The resulting narrative is a complex causal chain with many side arms contributing to the main causal sequence of events. It explains what happened and why it happened. Whether a narrative is accepted is assumed to depend on how well it accounts for all case-specific evidence, referred to as "coverage," the absence of alternative narratives, referred to as "uniqueness," and its "coherence" or logical consistency, completeness, and plausibility. If there is only one narrative with a high coverage and coherence, the decision maker should accept the narrative and be highly confident that the narrative represents what actually happened.

According to the story model, decision makers have to know how to classify narratives and to know how different types of narratives relate to actions. In the legal domain this knowledge is provided by the judge. To make a decision, a person has to construct a narrative and to classify the narrative as being of a particular type. The type of narrative, in turn, suggests the action to be taken. Depending on how well the constructed narrative matches the different types of narratives, decisions become 
more or less difficult. If a constructed narrative does not match any type or matches several types equally well, the narrative does not allow for a decision. Examples for this decision-making process can be found in the clinical domain. Illness narratives can be matched to illness scripts as outlined earlier or to descriptions in clinical guidelines that provide recommendations for treatment. For example, the NICE guideline on the treatment of depression in adults (CG90, NICE, 2009) explicitly states that "the choice of intervention should be influenced by [...] the trajectory of symptoms, previous course of depression and response to treatment." Depending on the type of development, different treatments are recommended. For example, antidepressant medication or highintensity psychological interventions (CBT or IPT) are recommended for people with mild to moderate depression who have not benefited from a low-intensity psychosocial intervention before.

Do causal knowledge and reasoning affect decision making based on narratives? The answer to this question seems trivial at first sight. Theories of narrative-based decision making, especially the story model (Pennington \& Hastie, 1992) consider causal knowledge to be a prerequisite for constructing a narrative. Domain-specific and generic causal knowledge about how the world works are assumed to be involved in constructing a narrative. However, a narrative may just capture the temporal, but not the causal sequence of events. Such a narrative may entirely depend on temporal knowledge, that is, knowledge about how things normally develop over time. For example, some illness scripts may only represent the typical course of a disease over time without any underlying causal mechanisms. To the best of our knowledge, there is no empirical research that directly investigated how causal and/or temporal knowledge affect the construction of narratives. There is some indirect evidence, however, from intercultural research on the explanation of illnesses (Furnham, 1988; Murdock, 1980). This research shows that generic assumptions about the factors that cause negative events affect how people explain the occurrence of an illness or accident. For example, the Azande believe that witchcraft is generally involved when negative events happen. In turn, their narratives explaining a person's illness involve witchcraft (Evans-Pritchard, 1976; see also Patel, 1995). Indirect evidence also comes from studies in the medical domain, which investigated how medical novices and experts represent diseases (cf. Schmidt \& Rikers, 2007). They indicate that illness scripts are used, which include knowledge about biomedical pathological mechanisms. 
By contrast, there is a lot of research that investigated how narratives affect decisions. The story model implies that decisions will be based on the narrative and not on the given information per se. Hence, different narratives provided for the same observations may result in different decisions. Evidence for this prediction comes from studies on legal decision making, which show that participants who generate different narratives to account for the same information, reach different verdicts (Pennington \& Hastie, 1986, 1988, 1992, 1993). The story model also predicts that a person's decision and confidence in the decision depend on coverage, coherence, and uniqueness of the constructed narrative. Several experiments by Pennington and Hastie (1992) showed that the coherence of the given information predicts decisions. The researchers also showed that information about causal relations affect decision makers' verdicts. Participants favored the verdict associated with the narrative for which information about causal links was explicitly provided. The influence of uniqueness upon jurors' decisions was investigated by Tenney, Cleary, and Spellman (2009). Participants in their study were highly sensitive to whether an alternative causal narrative could be constructed for the same evidence. When alternative narratives involving other suspects were pointed out, guilty verdicts dropped substantially.

The influence of narratives on decisions has also been investigated in the medical domain. These studies focused on patient decision making and explored whether providing people with a narrative would affect their choices. Winterbottom and colleagues conclude in a systematic review that narratives influence decisions more than statistical or general medical information (Winterbottom, Bekker, Conner, \& Montgomery, 2008). Decision maker's actions tended to mirror the actions of the person described in the narrative. However, an influence of narratives was found only in about a third of the studies.

In sum, there is good evidence that narratives influence decisions. They seem to be constructed spontaneously to organize given information into coherent causal chains, which explain how the current situation came about. These narratives are then used for decision making by identifying causes to target through interventions or by matching the narrative of a new case to remembered types of narratives that are related to specific actions.

\subsection{Decision Making Based on Cues}

Many decision-making strategies described in the literature rely on cues. It is beyond the aim of this chapter to provide a comprehensive review (see 
Koehler \& Harvey, 2004). We will focus on three exemplary strategies here, which have been described as relevant for clinical decision making (Groopman, 2007; Wegwarth, Gaissmeier, \& Gigerenzer, 2009). The first are fast and frugal heuristics (Gigerenzer, Todd, \& the ABC group, 1999). These heuristics assume that decision makers sequentially consider individual cues to make a choice among options (Gigerenzer \& Goldstein, 1996) or predict a criterion (Garcia-Retamero, Wallin, \& Dieckmann, 2007). When a cue favors a particular option over all other options, a decision in favor of that option is made, ignoring all further cues, which may have implicated other options. Cues are supposed to be considered according to their cue validity, that is, their relation to the criterion to be predicted (e.g., the severity of a disease, Garcia-Retamero et al., 2007). Although this strategy has not been described as a strategy to choose between actions that are supposed to lead to desired outcomes, it may be extended to cover these decisions as well. Depending on the present cues, different actions may have a different probability to result in the desired outcome. If this is the case, the observed cues will be predictive of the action to take. For example, to decide whether to give an antibiotic to a patient, a physician may check for fatigue, bodyand headaches, and the course of the symptoms, because these cues differentiate between viral and bacterial infections (National Institute of Health, 2015). Cues indicating a bacterial infection also indicate giving an antibiotic.

A second decision-making strategy based on cues is the usage of conditional rules, which specify the action to take if certain cues are present. Respective rules can be derived from observational and interventional learning (e.g., Colwill \& Rescorla, 1990; Enkvist, Newell, Juslin, \& Olsson, 2006; Juslin, Olsson, \& Olsson, 2003). In mental health, rules are usually not learned from feedback. Clinical guidelines provide the rules to practitioners. For example, the NICE guideline on PTSD describes the following rule to decide on an extended psychotherapeutic treatment (NICE, 2005). If symptoms of posttraumatic stress are present for more than 3 months and there are (1) multiple traumatic events, or (2) significant comorbid disorders, or (3) significant social problems, or (4) chronic disability resulting from the trauma, then extended trauma-focused psychotherapy (CBT or EMDR) should be considered.

The third decision-making strategy, which is also based on cues and relevant for decision making in the clinical domain, is decision making based on exemplars. One prominent theoretical model is recognition primed decision making (Klein, 1998), which assumes that cues observed in a specific case activate memories of similar previous cases stored in long 
term memory. Based on the similarity of the current and previous cases the action that led to the best outcome in similar cases in the past is chosen.

Do causal knowledge and reasoning affect decision making based on cues? A number of studies investigated how causal knowledge affects the search for cues and the weighting of cues in judgment and decision-making tasks (Garcia-Retamero \& Hoffrage, 2006; Garcia-Retamero et al., 2007; Mueller, Garcia-Retamero, Cokely, \& Maldonado, 2011; Mueller, Garcia-Retamero, Galesic, \& Maldonado, 2013). The findings show that participants prefer to search for cues indicating causal factors first. People also tend to weigh these cues more than other, equally valid cues that are not causes of the criterion to be predicted (Garcia-Retamero \& Hoffrage, 2006; Garcia-Retamero et al., 2007). Mueller et al. (2011, 2013) asked participants to decide which of two people was more likely to have a certain disease based on a set of cues representing risk factors for the disease. Cues also differed in their validity (i.e., how well they predicted the disease). In a series of experiments it turned out that participants' decisions were strongly affected by causal beliefs and less by the validity of the cue.

To the best of our knowledge, it has not been investigated whether and how causal knowledge affects decision making based on exemplars. Klein (1998) describes causal reasoning as an alternative to recognition primed decision making, when exemplars retrieved from memory do not allow the decision maker to choose an action. His research on naturalistic decision making indicates that decisions makers activate causal knowledge and simulate the consequences of potential courses of action when they cannot rely on similar previous cases (Zsambok \& Klein, 1997). These findings, however, do not address the question whether causal knowledge affects the selection of cues to activate similar cases in memory. There is some indirect evidence that it does. The studied experts focused on cues that were causally relevant for making a choice (e.g., experienced fire captains automatically checked for cues that indicate the structural integrity of a burning building, Klein, 1998).

\subsection{Do Causal Knowledge and Reasoning Improve Decision Making?}

The empirical findings summarized in the previous sections prove that causal considerations affect decision making. But do they improve decision making? Do causal knowledge and reasoning lead to decisions that generate better outcomes? We will start answering this question by providing a 
theoretical analysis exploring why causal knowledge and reasoning should result in better decisions. We will then discuss pertinent empirical findings.

There are several ways in which causal knowledge and reasoning may support decision making. First, causal knowledge may help to identify the cues that are relevant to consider when making a choice. To decide on an action, cues indicating causal factors that influence the likelihood of achieving the desired outcome are relevant. These factors include causes that maintain an undesired current state of affairs (e.g., a disease or disorder). An action will be effective to change the current state in a desired way if it addresses its causes. Hence, cues indicating problem-maintaining factors are relevant for decision making. Other relevant factors are moderators of an action's effectiveness. If a certain moderator is present an action may be less effective to generate the desired outcome. In extreme cases, negative instead of positive consequences may be caused by the same action. Thus, cues indicating moderators should be considered in choice. For example, a diabetic may feel dizzy because of a low or high blood glucose level. Depending on the cause different actions are implicated. Choosing the wrong action may be extremely dangerous for the person. Causal knowledge may also help decisions makers to identify the order in which cues should be considered. Cues strongly entailing a certain action should be taken into account before cues only moderately related to certain actions. Hence, cues indicating factors that strongly influence the effects and the effectiveness of the available options should be considered first. In the example of the diabetic the blood glucose level has to be the first cue to check for.

Causal knowledge also supports finding possible actions that lead to desired outcomes as it points out factors that influence the outcome and might be addressed by intervention. In the clinical domain, health could be the desired outcome, which has been defined as a state of complete physical, mental, and social well-being (WHO, 1946). In practice, a high global level of functioning or the absence of a currently present disorder is often taken as desired outcomes. Causal knowledge about the interventions and factors that affect a disorder and/or improve the level of functioning helps a decision maker to identify potential actions to be considered in decision making.

Causal knowledge may also help to identify the consequences that may result from an action. In clinical decision making, it is important to take into account not only desired outcomes of a treatment but also possible negative side effects. While very little is known about side effects of psychotherapeutic treatments, a lot is known about possible side effects of 
psychopharmacological treatments (e.g., for antidepressant medication see Brambilla, Cipriani, Hotopf, \& Barbui, 2005; Degner et al., 2004). By using such causal knowledge, a clinician is able to consider positive and negative outcomes that may result from a treatment.

Importantly, causal knowledge allows a decision maker to distinguish causal from spurious relations. As outlined earlier, expected utilities should be calculated based on probabilities that indicate causal, but not spurious relations. Actions that are only spuriously related to desired outcomes can be discarded straight away because choosing the action does not increase the probability of the desired outcome. As every action does involve some cost (e.g., time or effort) the utility of a spuriously related action would be lower than doing nothing. In clinical decision making, it is especially important to differentiate between elements of treatments that are effective and treatment elements that are not, although they correlate with desired outcomes, because they are combined with effective elements. For example, some antidepressant medications may have no positive effect that goes beyond a mere placebo effect, but have more negative side effects (Kirsch, Moore, Scoboria, \& Nicholls, 2002). Respective causal knowledge would allow a clinician to avoid these side effects.

Causal knowledge enables a decision maker to infer the probabilities of outcomes conditional on certain actions. As pointed out earlier, the probabilities to consider when deciding on an action are the probabilities of outcomes if the action would be deliberately chosen. These probabilities can be inferred from observed probabilities and the causal structure underlying these observations (Pearl, 2000; Sloman \& Lagnado, 2005; Waldmann \& Hagmayer, 2005). In the clinical domain, these probabilities are determined directly through randomized controlled trials (RCTs). RCTs are experimentally controlled studies, in which participating patients are randomly assigned to either a treatment or a control group. In consequence, the observed probabilities are a direct indicator of the probabilities that would result, if the same treatment would be chosen for the same type of patient. For example, the probability of a remission for patients suffering from PTSD is estimated to be between 50\% and 90\% when they receive a cognitive and/or behavioral intervention (see Bisson, Roberts, Andrew, Cooper, \& Lewis, 2013; for a review and metaanalysis). When clinicians want to use their clinical experience to derive such estimates, they would have to take into account possible confounds and common causes that result in spurious relations. To do so, they need causal knowledge. 
Causal knowledge in conjunction with observations allow a decision maker to infer the presence of not directly observable causal factors that may affect the outcome or that may moderate the effectiveness of an intervention. Causal expected utility theory assumes that the utility of an action depends on the probability of an outcome, which in turn depends on the action and the situation, and the probability of the situation (i.e., $\left.\operatorname{CEU}\left(\mathrm{A}_{\mathrm{i}}\right)=\sum_{\mathrm{ji}} P\left(\mathrm{O}_{\mathrm{ji}} \mid \mathrm{A}_{\mathrm{i}}, \mathrm{S}_{\mathrm{j}}\right) * P\left(\mathrm{~S}_{\mathrm{j}}\right) * \mathrm{U}\left(\mathrm{O}_{\mathrm{ji}}\right)\right)$. Hence, for a decision maker, it would be very helpful to know the situation $S_{j}$, that is, the conditions under which the decision is made. Diagnostic causal reasoning enables the decision maker to infer unknown aspect of the situation from observations (cf. Meder, Mayrhofer, \& Waldmann, 2014). In the clinical domain, it is important to know about the disorder(s) of the patient, because the type and severity of the disorder(s) may have a strong influence on the effectiveness of different interventions. For example, cognitive behavior therapy is known to be an effective treatment for mild to moderate depression, but a combination of psychotherapeutic and pharmacological treatment seems to be more effective for severe cases with psychotic symptoms (NICE, 2009). Causal knowledge about the symptoms that indicate these conditions enables the clinician to make inferences that will result in different estimates for the effectiveness of different treatments for a particular patient.

Given that causal knowledge and reasoning can support decision making in many ways, one would expect to find empirical evidence that they in fact lead to better outcomes. This is trivially true for many experimental studies on causal decision making cited earlier (e.g., Hagmayer \& Sloman, 2009). These experiments were designed in a way that considering causal structure leads to better choices by definition. Other studies were designed to show that causal knowledge may bias the weighting of cues in decision making (e.g., GarciaRetamero et al., 2007). None of these studies investigated decisions that people take outside the lab and that result in outcomes that matter to them.

Studies on patient decision making in health psychology provide such evidence. Studies found that the best predictors of patients' choices were their assumptions about the factors that control their illness (see Baines \& Wittkowski, 2013; Lobban, Barrowclough, \& Jones, 2003; for reviews). People who believe that professional treatments are able to control their illness are more likely to seek help and adhere to treatments. People who believe in personal control engage more in active coping and better health 
behavior (Baines \& Wittkowski, 2013; Lobban et al., 2003). Thus, different causal beliefs led to different actions. More important, causal beliefs also affected health outcomes. People with strong beliefs in personal and treatment control had a better prognosis than people who believed that their illness could not be controlled (Baines \& Wittkowski, 2013; Lobban et al., 2003). Thus, patients' causal assumptions could lead to choices that yield better outcomes.

To our surprise, there is very little empirical evidence on evidence-based practice in psychotherapy. Although clinicians are asked to take into account causal knowledge about the effectiveness of different treatments when deciding on a treatment plan for a particular patient, it is largely unknown whether doing so leads to significantly better outcomes for patients (cf. Kazdin, 2008). There is one (older) study, however, which shows that following an empirically evaluated treatment manual leads to a better outcome for patients than clinicians picking a treatment plan that they think is best (Schulte, Künzel, Pepping, \& Schulte-Bahrenberg, 1992).

Whether narratives improve decisions is not known at the moment. Winterbottom et al. (2008) conclude in their review on the effect of narratives on lay people's decisions in the medical domain that it is unclear whether narratives lead to better or worse choices. There is some indirect evidence from the medical domain, though. Expert clinicians have more elaborate illness scripts, which in turn improve diagnostic performance (Schmidt \& Rikers, 2007). A correct diagnosis of a patient's disease is a prerequisite for choosing an effective treatment. Hence, it seems likely that constructing a narrative and matching it to the scripts of different diseases may improve treatment choices and therefore the outcome for patients. The research by Pennington and Hastie (1992, 1993), however, shows that the construction of narratives is affected by many factors, including such arbitrary factors as the order in which the evidence is presented. Therefore, the constructed narrative may fail to be a valid representation of what actually happened when conditions are unfavorable (e.g., a mismatch between the actual order of events and the order in which the evidence was presented). It seems doubtful that decisions based on such invalid narratives lead to good outcomes.

In the clinical domain, a few studies investigated whether providing a causal explanation for a patient's condition results in better outcome for the patients (see Kuyken, Fothergill, Musa, \& Chadwick, 2005; Persons \& Hong, 2016; for overviews). These studies directly tested whether providing causal explanations has a positive utility for decision making. Litt, Kadden, 
and Kabela-Cormier (2009) investigated whether knowing about the situational factors that generate an urge to drink in people suffering from alcohol dependence would lead to better treatment outcomes. They found that knowing about these factors allowed clinicians to focus the treatment on high risk situations and the skills needed to cope with these situations. This in turn resulted in better coping and less drinking in comparison to a standardized treatment, which did not involve a causal analysis. Ghaderi (2006) also reported a positive utility of a functional causal analysis for clients suffering from bulimia nervosa. He showed that knowing about the influence of stimulus control, consequences, and discriminant stimuli on the patient's cognitions, emotions, and behaviors allowed clinicians to focus on effective interventions to address the identified causes. Other studies found no evidence that a causal analysis improved decisions in comparison to decisions based on an analysis of the symptoms to be addressed by treatment (e.g., Johansson et al., 2012).

\subsection{Summary}

The analysis presented in the previous sections showed that causal knowledge and reasoning have an effect on the decisions people make when they try to achieve a desired outcome. It also demonstrated that causal knowledge and reasoning may affect several different decision-making strategies, including strategies that do not presuppose that outcomes are considered in decision making. Cue-based decision-making strategies are a prime example of the latter.

The analysis also indicated that causal knowledge and reasoning may improve the choices made. More precisely, causal knowledge and reasoning can make it more likely that people pick the option that raises the probability of the desired outcome the most. They do, because they allow decision makers (1) to avoid actions that are only spuriously related to the outcome, (2) to consider factors that may moderate the effectiveness of an action, and (3) to target the factors whose changes will have the highest impact on the desired outcome. But the existing evidence on the utility of causal considerations in clinical decision making is mixed. Causal knowledge and reasoning sometimes seem to improve decisions and the outcomes that result from them, but they sometimes seem to make no difference. In principle, they might even be detrimental, when causal beliefs lead to misjudgments of efficacy.

Therefore, the question is when and how causal knowledge and reasoning should be used in decision making to get better outcomes. In 
the second part of this chapter, we will propose an answer and describe a framework based on the existing evidence and theoretical analyses.

\section{INTEGRATING CAUSAL KNOWLEDGE AND REASONING INTO DECISION MAKING-THE CAUSAL- EXPLANATION-BASED DECISION-MAKING FRAMEWORK}

In line with the theoretical analyses presented earlier, we assume that causal knowledge and reasoning can and-under appropriate conditionswill improve decisions in a way that leads to better outcomes. We propose a five-step process, of which the first step is to decide whether to use causal knowledge or not. Thus, we do not assume that using causal knowledge will always lead to better decisions. The next steps spell out in more detail how causal knowledge should be used and which inferences have to be made. The final step describes how observations of outcomes should be used to keep or revise decisions. Fig. 1 visualizes the five-step process. We call our framework the CDM, because it involves a causal explanation of the current situation that the decision maker intends to change.

Our proposal is inspired by prescriptive models of decision making in the clinical domain, which recommend a causal analysis of the factors and processes that led to an individual patient's problem and maintain it (e.g., Eells, 2007; Haynes, Leisen, \& Blaine, 1997; Kuyken, 2006; Persons, 2008), and insights from the research on causal reasoning and decision making (e.g., Hagmayer \& Sloman, 2009).

The starting point of the decision-making process is a problem. Usually it is an event or state that is either undesired (has negative utility) in itself or has undesired consequences. In principle, it may be any situation, in which a decision maker is searching for actions to improve a current state of affairs. In clinical decision making (in mental and physical health) the starting point is a patient presenting with symptoms and behavioral problems, who is suffering from her or his condition and desires to reduce the suffering by reducing the symptom load.

\subsection{Step 1: Decide on Causal Analysis}

The goal of the first step is to check whether a causal explanation of the problem is likely to improve the decision on the action to take. Thus, it needs to be determined whether knowing about the causal factors and mechanism that contributed and still contribute to the problem would result 


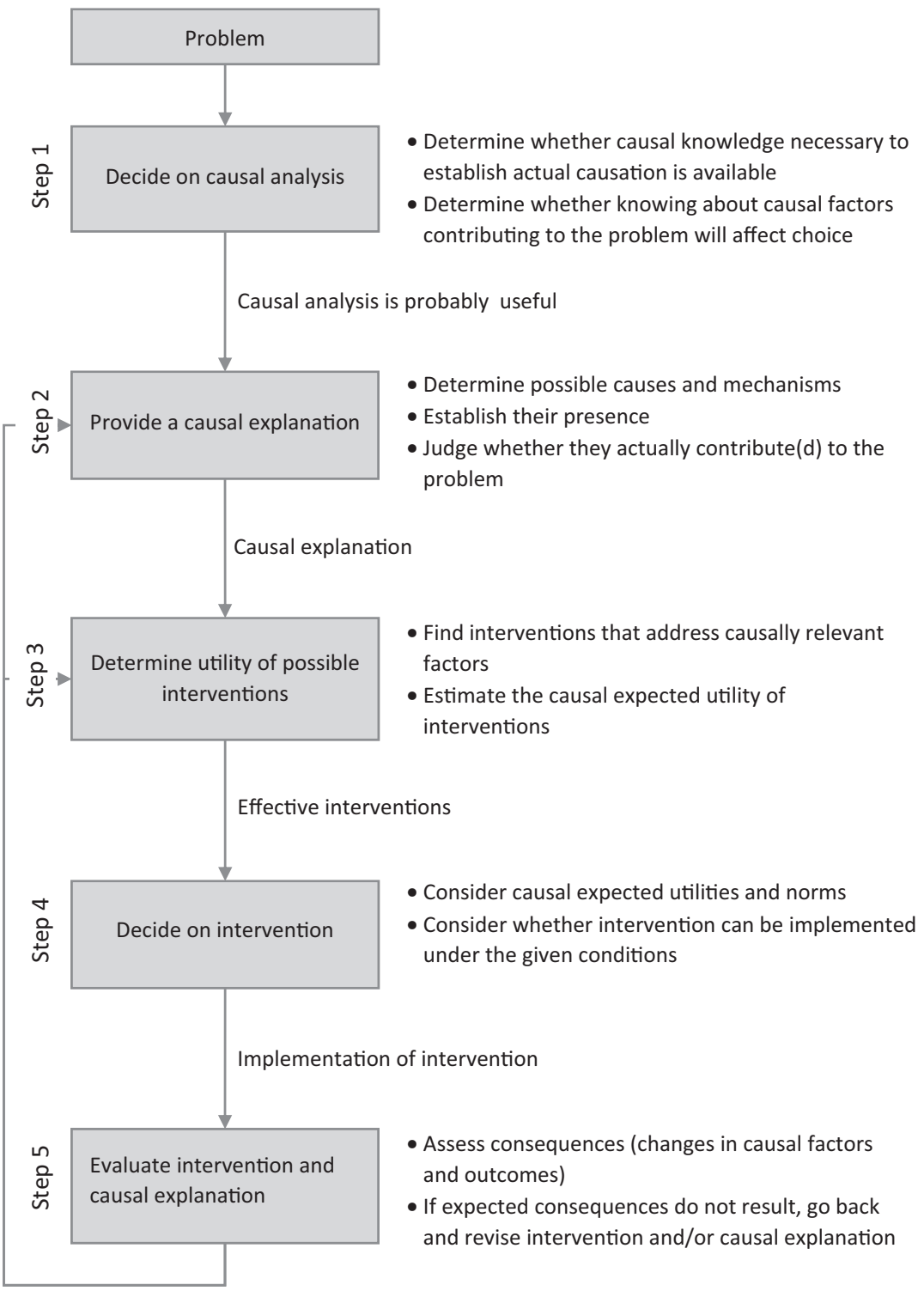

Figure 1 Causal-explanation-based decision making framework. See text for a full description of the five-step process.

in a different choice that is likely to lead to a better outcome. Sometimes there is only one effective option, which makes further causal analyses superfluous. If there are several courses of action to choose from and there is time to deliberate, two sets of preconditions for the usefulness of a causal 
explanation in decision making should be checked. The first set comprises conditions for being able to provide a causal explanation. The second set specifies conditions for the utility of a potential explanation in decision making.

Although there is no universally agreed definition of what it means to provide an explanation, there seems to be some consensus (cf. Keil, 2008; Lombrozo, 2006). To give an explanation involves identifying the causal factors and mechanisms that were present and actually generated the problem to be explained and/or the problem's function (its final cause). To do so, the decision maker needs valid, generic causal knowledge about the factors generating and maintaining the type of problem at hand and the mechanisms by which the factors affect the type of problem. In addition, the decision maker has to know how to assess whether the potentially relevant factors are present and whether present factors actually affected the problem. In brief, a decision maker needs the causal knowledge required to establish actual causation.

In clinical decision making in mental health, this knowledge is provided by theories of psychopathology, which describe factors and mechanisms that may lead to specific problematic behaviors and symptoms (see for example, Maddux \& Winstead, 2016). These theories also describe the function that the symptoms serve for the affected person. In general, valid and reliable tools are available to assess causally relevant factors. These tools include structured diagnostic interviews and psychological tests.

Even when a decision maker has the knowledge required for providing a causal explanation, it might not make sense to search for the explanation. If finding the best option only depends on knowing the problem and its features, there is no need for a causal explanation. By contrast, searching for a causal explanation is indicated if the available options are interventions that target specific factors (and mechanisms) that cause and maintain the present type of problem. In this case, it would be useful to know which causal factors actually contribute to the problem to be able to pick the intervention(s) that address these factors. Therefore, important conditions for the utility of causal explanation in decision making are that there are interventions addressing the causes of a problem and that the decision maker knows how the interventions affect these causal factors. Just knowing that the interventions effectively address the problem is not sufficient for a causal explanation to be useful to make a choice.

If the decision maker is able to provide a causal explanation and a causal explanation is likely to have a positive utility to choose an effective 
intervention, then the decision maker should move on to Step 2 of the process. If the conditions are not met, the decision maker should choose an intervention that proved to be effective to resolve the same type of problem in the past. In the clinical domain, this would mean that the clinician should choose the most effective, empirically supported treatment for the disorder(s) presented by the patient.

\subsection{Step 2: Provide a Causal Explanation for the Problem}

The goal of the second step is to come up with a causal explanation of the problem, that is, to identify the causal factors and mechanisms that actually generated and maintain the particular problem at hand. Only if the causes actually contributing to the problem are known, interventions can be chosen that change these factors and mechanisms.

To provide an explanation, first the potential causes have to be identified. Possible causes of a type of problem can be retrieved from memory or looked up in knowledge bases (e.g., textbooks). Research on causal attribution has shown that people automatically start to think about potential causes of events and states that are negative or unexpected and retrieve relevant knowledge from memory (Kahneman \& Miller, 1986; Weiner, 1985).

Next, it has to be established whether these causes were or are still present. Sometimes the factors or mechanisms can be directly observed, but sometimes their presence has to be inferred from features of the problem or other observable indicators. Research on diagnostic reasoning has shown that participants are quite good at inferring the probability of a cause being present from the presence of its effects (cf. Meder et al., 2014). Interestingly, inferences tend to take into account uncertainties about the causal relation, its strength, and at least sometimes the base rate of the cause to be inferred (Krynski \& Tenenbaum, 2007). Corroborating evidence comes from research investigating the questions people ask when they are requested to provide an explanation. Studies found that people often inquire about factors that they know to be causally related to the type of event to be explained (Ahn, Kalish, Medin, \& Gelman, 1995).

Finally, it has to be inferred whether the causes that are known to be present or are inferred to be present, actually affected the problem. Even when a cause influences an event in general, it may have had no influence in a particular case. How people make inferences about actual causation in singular instances is a currently much researched topic (cf. Danks, 2016). Findings indicate that people believe in actual causation when a connecting mechanism is directly observable, but they seem to rely on counterfactual 
dependence, when there is no perceivable mechanism (Walsh \& Sloman, 2011). Omitted actions cannot be related to an outcome through a mechanism. Nevertheless, an omission is considered to be an actual cause of an event if the event would not have happened if the action was not omitted (Lombrozo \& Vasilyeva, 2016). For example, not providing an acute psychiatric intervention to a suicidal patient is judged to be a cause of the patient's suicide, because the intervention would have prevented the suicide if it had been provided. Finally, Stephan and Waldmann (2016) showed that people take into account the sufficiency of a cause for its effect and the uncertainty about generic causation when judging actual causation. To put it simply, reasoners consider how often a causal factor leads to an effect in general and how much evidence there is for this causal mechanism. Thus, people seem to be able to infer actual causation even in cases in which there is neither an observable mechanism nor counterfactual dependence.

Providing a causal explanation is part of developing a clinical case formulation. According to Persons and Hong (2016) a comprehensive case formulation includes a description of the patient's problems and symptoms, the factors and mechanisms that cause and maintain the problems, and the features of the patient or environment that are likely to affect the treatment outcome (see also Eells, 2007; Tarrier \& Johnson, 2016). Clinicians may explain a patient's problems in several ways. Some explain a problem by describing the process through which the problem developed over time, that is, they construct a narrative. Such a narrative is usually based on illness scripts and theories of psychopathology as well as the patient's history. Others may explain the problems by pointing out factors and mechanisms that are likely to have causally contributed to the patient's problems (e.g., Haynes et al., 1997). Potentially relevant causal factors and mechanisms are described in etiological theories and summarized in a biopsychosocial model of a particular disorder. Which of these factors are present is determined by diagnostic tests and a clinical interview. Currently there are no clear criteria on how to establish actual causation in the clinical domain. Some seem to assume that the presence of a causal factor is sufficient for its actual contribution (e.g., Haynes et al., 1997). Others take the covariation of a factor with the behavioral problems of the particular patient as an indicator (e.g., Litt et al. 2009). The mutual agreement between client and therapist that a factor actually contributes to the problem may also be used as an indicator (Kuyken, 2006).

There are, however, doubts about the reliability of providing a causal explanation by a narrative or a summary of present causal factors 
(Garb, 2005; Kuyken et al., 2005). Findings indicate that clinicians only moderately agree about the causal explanation of a patient's problems (DeKwaadsteniet et al., 2010; Witteman \& Koele, 1999). The only moderate agreement may be due to the many different ways in which clinicians may explain a patient's problems. Studies focusing on a particular type of causal explanation of behavioral problems (functional analyses in cognitive behavior therapy) found a much better reliability across clinicians (Ghaderi, 2007).

Once a causal explanation is provided, the decision maker should move on to Step 3. But, Step 2 may fail even when the preconditions for providing a causal explanation are met. It may turn out that none of the known causes of the problem are present or that there is no evidence indicating that the present factors are the actual causes. In this case, the decision maker should choose an intervention that is known to be effective for the type of problem rather than relying on the causal explanation.

\subsection{Step 3: Determine the Utility of Possible Interventions}

The goal of the third step is to identify the intervention or set of interventions that will change the factors and mechanisms that actually generated and maintain the problem and to estimate how effective these interventions are to address the problem. More technically, the goal of Step 3 is to determine the causal expected utility of possible interventions and rank them accordingly.

To do so, first potential interventions have to be retrieved from memory or other knowledge bases. Note that the causal explanation provided in Step 2 constrains the search by identifying the actual causes, which should be targeted by the intervention. Danks (2016) suggests a heuristic, which first searches for interventions that directly address the undesired aspects of a problem, then moves on to interventions that address immediate causes of the problem before searching for interventions that address indirect, distal causes of the problem. Once an intervention is found that would eliminate the problem, the search is stopped. Simulation studies showed that this heuristic greatly simplifies the search for effective interventions (Danks, 2016).

Once potential interventions have been identified, the consequences of implementing these interventions have to be inferred. Speaking more technically, the CEU of the different options have to be estimated. By representing the current problem as a causal model and possible interventions as exogenous factors in these models, decision makers can infer the consequences and their likelihood (Danks, 2016; Sloman \& Hagmayer, 2006). As pointed out in the first part of this chapter, decision makers are able to 
derive these inferences (Hagmayer \& Sloman, 2009; Robinson et al., 2010). They can identify the most effective and therefore most useful intervention, at least when the causal structure is rather simple and the problem does not change dynamically over time (Hagmayer \& Meder, 2013; Hagmayer et al., 2010).

In the clinical domain, practitioners are advised to consider the evidence regarding the effectiveness of different types of interventions when deciding on the treatment of an individual patient (APA Presidential Task Force on Evidence-Based Practice, 2006). Proponents of clinical case formulations, by contrast, suggest using a client-specific case formulation to judge the effectiveness of different interventions (e.g., Haynes et al., 1997; Persons, 2008). There is some empirical evidence that the causal explanation put forward by a clinician affects how this clinician judges the effectiveness of available interventions for a particular client. DeKwaadsteniet et al. (2010) asked clinicians to create a causal model explaining the problems of two children who showed symptoms of behavioral and/or mood disorders. Clinicians also had to rate the effectiveness of common treatments for these two children. In addition, they were asked which factors were targeted by these treatments. The results showed that clinicians' individual causal models predicted their effectiveness ratings quite well.

Once the available interventions are ranked depending on their effectiveness for achieving the desired outcomes (i.e., their causal expected utility) the decision maker moves on to Step 4. It may turn out that several interventions have roughly the same causal expected utility. Especially in mental health, several types of treatments have been shown to be equally effective to achieve a remission. For example, there seem to be no significant differences between cognitive behavior therapy, exposure therapy, and EMDR (eye movement desensitization and reprocessing) when treating PTSD (Bisson et al., 2013). Step 4 addresses this issue.

\subsection{Step 4: Decide on an Intervention}

The goal of this step is to choose an intervention to address the given problem. One obvious possibility is to choose the option that has the highest causal expected utility (principle of maximizing causal expected utility, Nozick, 1993). By choosing this option, the decision maker picks the option that has the highest likelihood of achieving the most desired outcome. However, a decision maker may prefer not to follow the principle of maximizing causal expected utility. If the decision maker's goal is to minimize the risk of a negative outcome, she should 
maximize the minimal utility (maximin principle, Wald, 1945). A multitude of different choice rules have been described in the literature (cf. Resnik, 1987). Which choice rule should be used from a normative point of view depends on the domain. In medicine, for example, the maximin principle may be preferred for frail patients, because it prevents harming the patient. A decision based on the maximin principle is in line with the "first, do no harm principle" (primum non nocere), which is an important principle in medical ethics.

Apart from considerations of causal expected utility, there are norms (moral, social, and legal) that come into play at this point. These norms should be taken into account as well. In the clinical domain, decision making is regulated by organizational, professional, and legal norms and guidelines. These prescriptions may conflict with each other. For example, evidence-based guidelines in the United Kingdom prescribe that a patient suffering from bulimia nervosa (i.e., an eating disorder characterized by the patient throwing up frequently) should be treated by means of an evidence-based self-help manual before transferring the patient into an outpatient treatment program offering cognitive behavior therapy (NICE, 2004). For a particular patient, though, a causal analysis may indicate that she should be transferred to an inpatient facility, because the habitual unhealthy eating patterns cannot be effectively changed through self-help manuals or outpatient treatment. In this case, the causal expected utility would be highest for a transferal to inpatient treatment, but the decision maker may decide in favor of outpatient treatment following the clinical guideline.

In the domain of mental and physical health, there is another challenge, as the effectiveness of an intervention may depend on how the decision is made (cf. Simon, Wills, \& Harter, 2009). If a patient does not commit to a treatment, it will be less effective than when she or he does commit to it. The patient's commitment in turn may depend on how they are involved in the decision. Involving the patient into the decision may show that the patient's goals and preferences are not compatible with the treatment that the clinician assumes to have the highest causal expected utility. In this case, the clinician needs go back to Step 3 and reconsider the assumptions made about the effectiveness of different treatments taking into account the patient's preferences.

Once a decision is made, the chosen intervention should be implemented, and the decision maker should move on to Step 5. If the chosen option cannot be implemented, Step 4 needs to be reiterated to identify the next best option. 


\subsection{Step 5: Evaluate the Intervention and Causal Explanation}

The goal of Step 5 is to find out whether the expected consequences result from the chosen intervention. If the causal explanation of the problem is correct, then interventions on the causal factors that generated and maintain the problem should result in positives outcomes.

To evaluate the intervention and the causal explanation, the problem and its causal factors and mechanisms need to be reassessed on a regular basis. This will show whether the interventions change the factors and mechanisms as expected. It will also show whether these changes reduce and finally solve the problem. If the interventions turn out to be ineffective and the problem remains the same, the decision maker should go back to Step 3 and find new interventions that address the actual causes of the problem. If the interventions prove to be effective in changing the factors they are supposed to address, but the problem does not change, then the causal explanation is probably wrong. In this case, the decision maker has to go back to Step 2 and reconsider the causal explanation. As described earlier, determining which factors are the actual causes of a present problem is often the most difficult inference to make. Therefore, assumptions about the actual contribution of different factors might be wrong.

As far as we know, no experimental research in cognitive psychology has been conducted investigating if and how people evaluate an intervention and the causal explanation upon which the decision was based when they are presented with information on the resulting consequences. There are quite a number of studies, though, investigating how learners change their assumptions about causal structure when new evidence arrives (e.g., Fernbach \& Sloman, 2009; Steyvers, Tenenbaum, Wagenmakers, \& Blum, 2003). These studies, however, do not deal with learning about a single instance. The same is true for research on repeated causal decision making (Hagmayer \& Meder, 2013). Research on the story model of decision making (Pennington \& Hastie, 1992) shows that people take newly arriving information into account when providing a causal narrative for a particular instance. But these studies have not investigated what happens to the causal explanation when people are informed about the causal consequences of the decision being made.

In the clinical domain, the evaluation of treatment progress is an important issue. Symptoms, suffering, and subjective progress are often evaluated on a regular basis using standardized assessment tools (cf. Shimokawa, Lambert, \& Smart, 2010). The same seems to be true for at least some causal 
factors and mechanisms. For example, the frequency of avoidant behaviors and dysfunctional coping in the treatment of phobic disorders can be checked through self-monitoring. Proponents of the usage of clinical case formulations advise to make such systematic assessments throughout treatment to evaluate and-if necessary-to revise the case formulation (Persons \& Hong, 2016). Research showed that collecting information on treatment progress results in better treatment outcomes (Knaup, Koesters, Schoefer, Becker, \& Puschner, 2009). At present, however, it is unclear whether this positive effect is due to changing elements of chosen interventions, switching to new interventions targeting the same causes, redirecting interventions to other causal factors, or coming up with a new causal explanation for a patient's problem.

\subsection{Summary}

The five-step process of CDM describes when and how a decision maker should use a causal explanation to make a decision. If the two conditions specified in Step 1 are met, then a decision based on the causal explanation is likely to result in a better outcome than a decision that is not based on a causal explanation. These two conditions check that a causal explanation can be provided and that the effectiveness of an intervention will depend on the factors identified in the explanation. When decision makers follow the prescribed process they will choose interventions that are likely to target the actual causes of the problem to be resolved. By targeting the actual causes, the chosen interventions should be more effective than interventions that merely address likely causes of the type of problem. In the latter case, interventions may be implemented that address factors that are not actually present, do not need to be changed, or make no difference in the particular case. Thus there is a good rationale to assume that the five-step process may result in choices that lead to better outcomes than ways of decision making that do not consider causal explanations. Whether it actually does, needs to be shown through empirical research.

\section{CONCLUSIONS}

Our goals for this chapter were to analyze how causal knowledge and reasoning affect, support and potentially improve decision making in a way that leads to better outcomes. A review of the empirical research on the role of causal knowledge and reasoning showed that they affect decision making. 
They influence not only decision making based on expected outcomes and narratives, but also decision making based on cues. A theoretical analysis showed that there are good reasons to assume that causal knowledge and reasoning improve decision making. Decisions makers who base their decisions on causal considerations should be more likely to choose the action that increases the likelihood of the desired outcome the most and eventually results in the desired outcome most often. A review of studies that empirically investigated the influence of causal considerations on outcomes in the clinical domain, however, showed that rather little is known at the moment. Although some studies found a positive influence, others found no effect. This is surprising, because clinicians are advised to take causal factors into account to provide better care. Even evidence-based guidelines recommend analyzing specific causal factors and the development of a patient's problems over time.

The heterogeneous findings with respect to outcomes might be due to the fact that causal considerations lead to better outcomes only under certain conditions. We pointed out two conditions: (1) decisions makers have to have relevant knowledge about causes and interventions and (2) the efficacy of the actions to choose from has to depend on causal factors that can be considered in decision making. If these conditions are met, causal considerations are likely to improve decisions; if the conditions are not met, no positive effect has to be expected.

Based on the theoretical analyses in the first part of the paper, case formulation approaches to clinical decision making, and insights from the research on causal decision making, we proposed the CDM. It describes when and how causal knowledge and reasoning should be used in decision making to achieve better outcomes. Its first step is to check whether the two conditions specified earlier are met. The second step is to provide a causal explanation for the problem that should be changed for the better. The third step is to use the causal explanation to find and judge possible interventions for their effectiveness to generate a desired outcome. Step 4 is to make a choice based on CEU and implement the chosen action. The final step is to monitor the consequences resulting from the choice and-if expected consequences do not result-revise the decision and the causal explanation. There is evidence that decision makers have the competence to perform every step of the proposed process. We hypothesize that following these steps will allow decision makers to use causal considerations when they improve decisions and maximize the utility of the chosen actions. 
At present, the CDM framework is a blueprint. It is a blueprint for a future rational, prescriptive model of decision making, at least in the clinical domain. A rational model of treatment choice would describe how a rational decision maker would use clinical causal knowledge to choose a treatment to improve the outcome of a patient (cf. Anderson, 1990). To turn the CDM framework into a rational model, several things need to be done. First, a computational model needs to be developed, which implements the proposed process. Causal Bayes nets (Pearl, 2000; Spirtes, Glymour, \& Scheines, 2000) are an excellent starting point (see Chater \& Oaksford, 2008; Danks, 2016; Tenenbaum et al., 2011). Causal Bayes nets allow us to model causal knowledge, inferences based on causal knowledge, and decisions based on CEU. It will be a challenge, though, to implement the complex and extensive knowledge of clinicians (including illness scripts, knowledge about guidelines, evidence-based treatments, and domainspecific choice rules) as a causal Bayes net.

Once the model is implemented, it will be possible to run simulations. These simulations will show whether the model will lead to better outcomes and whether the proposed conditions for engaging in causal considerations are effective to prevent decision makers from using causal knowledge when it does not lead to better outcomes. It will also be possible to investigate whether the proposed process is robust, that is, whether it works well under a multitude of conditions. Knowledge about the causes and mechanisms that generate and maintain mental disorders is still uncertain, as is the knowledge about the mechanisms by which interventions work (Carr, 2009; Kazdin, 2008). The CDM model should work well under these conditions. Apart from simulations, it will be important to show that the five-step process leads to better outcomes in clinical practice. This will be the ultimate test to prove that it is rational to follow its recommendations.

Rational models in the sense of Anderson (1990) are also supposed to be descriptive of people's performance, when they are adapted to the task. Therefore, rational models have often sparked very productive lines of research. Causal Bayes nets and the research on causal learning and reasoning in the last 20 years is a good example (see Gopnik \& Schulz, 2007; Waldmann, 2016). The CDM framework could be a rich source of hypotheses. We want to point out some hypotheses that we think would be worthwhile to test. Step 1 prescribes that decision makers should only use causal knowledge and engage in a causal analysis when it is likely that it allows them to make a better decision. How decision makers proceed, should therefore depend on their generic causal knowledge about causal 
factors and interventions. Step 2 prescribes that decision makers should come up with an explanation that focuses on the causal factors that actually make a difference for the problem to be resolved. This implies that explanations should differ in a predictable way from generic causal models representing all the factors that may contribute to the type of problem. Step 3 prescribes that only interventions directly addressing the problem or its actual causes should be considered when deciding on action. This entails that different interventions should be considered depending on the specific case. Step 3 also prescribes that decision makers should estimate the CEU of the different options. Step 4 prescribes that CEU should be taken into account when a choice is made. Hence, decisions should depend on these utilities. Step 5 prescribes that a decision maker should not only assess resulting outcomes, but also the factors that would be changed if the chosen intervention had the expected effect. Depending on whether the targeted causal factors have changed the intervention or the causal explanation should be revised. Many more interesting hypotheses could be derived from more specific assumptions about the causal structures underlying the problem to be addressed and the knowledge the decision maker has about these structures.

If it turns out that the predictions derived from the CDM framework are supported by the empirical evidence, we may have found a rational model for clinical decision making that involves the adaptive usage of causal knowledge. If hypotheses are not supported, we will at least have gained many new insights on when and how causal knowledge and reasoning affect decision making and lead to better outcomes.

\section{REFERENCES}

Ahn, W.-K., Kalish, C. W., Medin, D. L., \& Gelman, S. A. (1995). The role of covariation versus mechanism information in causal attribution. Cognition, 54, 299-352.

Ahn, W.-K., Proctor, C. C., \& Flanagan, E. H. (2009). Mental health clinicians' beliefs about the biological, psychological, and environmental bases of mental disorders. Cognitive Science, 33, 147-182.

American Psychiatric Association. (2000). DSM-IV-TR: Diagnostic and statistical manual of mental disorders (4th ed., rev.). Washington, DC: Author.

Anderson, J. R. (1990). The adaptive character of thought. Hillsdale, NJ: Erlbaum.

APA Presidential Task Force on Evidence-Based Practice. (2006). Evidence-based practice in psychology. American Psychologist, 61, 271-285.

Baines, T., \& Wittkowski, A. (2013). A systematic review of the literature exploring illness perceptions in mental health utilising the self-regulation model. Journal of Clinical Psychology in Medical Settings, 20, 263-274.

Barlow, D. H. (2002). Anxiety and its disorders: The nature and treatment of anxiety and panic. New York: Guilford Press. 
Bisson, J. I., Roberts, N. P., Andrew, M., Cooper, R., \& Lewis, C. (2013). Psychological therapies for chronic post-traumatic stress disorder (PTSD) in adults. Cochrane Database of Systematic Reviews, 12, CD0033.

Bodner, R., \& Prelec, D. (2003). The diagnostic value of actions in a self-signaling model. The Psychology of Economic Decisions, 1, 105-123.

Brambilla, P., Cipriani, A., Hotopf, M., \& Barbui, C. (2005). Side-effect profile of fluoxetine in comparison with other SSRIs, tricyclic and newer antidepressants: A meta-analysis of clinical trial data. Pharmacopsychiatry, 38, 69-77.

Carr, A. (2009). The effectiveness of family therapy and systemic interventions for childfocused problems. Journal of Family Therapy, 31(1), 3-45.

Charlin, B., Boshuizen, H. P., Custers, E. J., \& Feltovich, P. J. (2007). Scripts and clinical reasoning. Medical Education, 41, 1178-1184.

Chater, N., \& Oaksford, M. (2008). The probabilistic mind. Prospects for Bayesian cognitive science. Oxford: Oxford University Press.

Colwill, R. M., \& Rescorla, R. A. (1990). Evidence for the hierarchical structure of instrumental learning. Animal Learning and Behavior, 18, 71-82.

Danks, D. (2014). Unifying the mind. Cognitive representations as graphical models. Cambridge, MA: MIT Press.

Danks, D. (2016). Singular causation. In M. R. Waldmann (Ed.), Oxford handbook of causal reasoning. Oxford: Oxford University Press.

Degner, D., Grohmann, R., Kropp, S., Ruther, E., Bender, S., Engel, R. R., \& Schmidt, L. G. (2004). Severe adverse drug reactions of antidepressants: Results of the German multicenter drug surveillance program AMSP. Pharmacopsychiatry, 37(Suppl. 1), 39-45.

DeKwaadsteniet, L., \& Hagmayer, Y. (2017). Clinicians' personal theories of developmental disorders explain their judgments of effectiveness of interventions (Manuscript submitted for publication).

DeKwaadsteniet, L., Hagmayer, Y., Krol, N., \& Witteman, C. (2010). Causal client models in selecting effective interventions: A cognitive mapping study. Psychological Assessment, 22, 581-592.

Dozois, D. J., Mikhail, S. F., Alden, L. E., Bieling, P. J., Bourgon, G., Clark, D. A., ... Johnston, C. (2014). The CPA presidential task force on evidence-based practice of psychological treatments. Canadian Psychology, 55(3), 153-160.

Eells, T. D. (2007). Handbook of psychotherapy case formulation (2nd ed.). New York, NY: Guilford Press.

Enkvist, T., Newell, B., Juslin, P., \& Olsson, H. (2006). On the role of causal intervention in multiple cue judgment: Positive and negative effects on learning. Journal of Experimental Psychology: Learning, Memory, and Cognition, 32, 163-179.

Evans-Pritchard, E. E. (1976). Witchcraft, oracles and magic among the Azande. Oxford: Oxford University Press.

Feltovich, P. J., \& Barrows, H. S. (1984). Issues of generality in medical problem solving. In H. G. Schmidt, \& M. L. De Voider (Eds.), Tutorials in problem-based learning: A new direction in teaching the health professions. Assen, Holland: Van Gorcum.

Fernbach, P. M., Darlow, A., \& Sloman, S. A. (2010). Neglect of alternative causes in predictive but not diagnostic reasoning. Psychological Science, 21, 329-336.

Fernbach, P. M., \& Sloman, S. A. (2009). Causal learning with local computations. Journal of Experimental Psychology: Learning, Memory, and Cognition, 35, 678-693.

Fernbach, P. M., Sloman, S. A., \& Hagmayer, Y. (2014). Effort denial in self-deception. Organizational Behavior and Human Decision Processes, 123, 1-8.

Flores, A., Cobos, P. L., López, F. J., \& Godoy, A. (2014). Detecting fast, on-line reasoning processes in clinical decision making. Psychological Assessment, 26, 660-665. 
Flores, A., Cobos, P. L., López, F. J., Godoy, A., \& González-Martín, E. (2014). The influence of causal connections between symptoms on the diagnosis of mental disorders: Evidence from on-line and off-line measures. Journal of Experimental Psychology: Applied, 20, 175-190.

Furnham, A. (1988). Lay theories. Everyday understanding of problems in the social sciences. Oxford: Pergamon Press.

Garb, H. N. (2005). Clinical judgment and decision making. Annual Review of Clinical Psychology, 1, 67-89.

Garcia-Retamero, R., \& Hoffrage, U. (2006). How causal knowledge simplifies decisionmaking. Minds and Machines, 16, 365-380.

Garcia-Retamero, R., Wallin, A., \& Dieckmann, A. (2007). When one cue is not enough: Combining fast and frugal heuristics with compound cue processing. Quarterly Journal of Experimental Psychology, 60, 1197-1215.

Ghaderi, A. (2006). Does individualization matter? A randomized trial of standardized (focused) vs. individualized (broad). Cognitive behavior therapy for bulimia nervosa. Behaviour Research and Therapy, 44, 273-288.

Ghaderi, A. (2007). Logical functional analysis in the assessment and treatment of eating disorders. Clinical Psychologist, 11, 1-12.

Gigerenzer, G., \& Goldstein, D. G. (1996). Reasoning the fast and frugal way: Models of bounded rationality. Psychological Review, 103, 650-669.

Gigerenzer, G., Todd, P., \& ABC Group. (1999). Simple heuristics that make us smart. New York: Oxford University Press.

Gopnik, A., \& Schulz, L. (2007). Causal learning: Psychology, philosophy, and computation. Oxford, England: Oxford University Press.

Groopman, J. (2007). How doctors think. Boston: Houghtom \& Mifflin.

Hagmayer, Y., \& Meder, B. (2013). Repeated causal decision making. Journal of Experimental Psychology: Learning, Memory, and Cognition, 39, 33-50.

Hagmayer, Y., Meder, B., Osman, M., Mangold, S., \& Lagnado, D. (2010). Spontaneous causal learning while controlling a dynamic system. The Open Psychology Journal, 3, 145-162. Retrieved from http://www.benthamscience.com/open/topsyj/articles/ V003/SI0088TOPSYJ/145TOPSYJ.pdf.

Hagmayer, Y., \& Sloman, S. A. (2009). People conceive of their choices as intervention. Journal of Experimental Psychology: General, 138, 22-38.

Haynes, S. N., Leisen, M. B., \& Blaine, D. D. (1997). Design of individualized behavioral treatment programs using functional analytic clinical case models. Psychological Assessment, 9, 334-348.

Huber, O., Huber, O. W., \& Bär, A. S. (2011). Information search and mental representation in risky decision making: The advantages first principle. Journal of Behavioral Decision Making, 24, 223-248.

Huber, O., Wider, R., \& Huber, O. W. (1997). Active information search and complete information presentation in naturalistic decision tasks. Acta Psychologica, 95, 15-29.

Johannson, R., Sjöberg, E., Sjögren, M., Johnsson, E., Carlbring, P., Andersson, T., ... Andersson, G. (2012). Tailored vs. standardized internet-based cognitive behavior therapy for depression and comorbid symptoms: A randomized controlled trial. PLoS One, 7(5), e36905. http://dx.doi.org/10.1371/journal.pone.0036905.

Joyce, J. M. (1999). The foundations of causal decision theory. Cambridge, UK: Cambridge University Press.

Juslin, P., Olsson, H., \& Olsson, A. C. (2003). Exemplar effects in categorization and multiple-cue judgment. Journal of Experimental Psychology: General, 132, 133-156.

Kahneman, D. (2011). Thinking fast and slow. London: Penguin Books.

Kahneman, D., \& Miller, D. T. (1986). Norm theory: Comparing reality to its alternatives. Psychological Review, 93, 136-153. 
Kahneman, D., \& Tversky, A. (1979). Prospect theory: An analysis of decision under risk. Econometrica, 47, 263-291.

Kazdin, A. E. (2008). Evidence-based treatment and practice: New opportunities to bridge clinical research and practice, enhance the knowledge base, and improve patient care. American Psychologist, 63, 146-159.

Keil, F. C. (2008). Explanation and understanding. Annual Review of Psychology, 51, 227-254.

Kim, N. S., \& Ahn, W. (2002). Clinical psychologists' theory-based representations of mental disorders predict their diagnostic reasoning and memory. Journal of Experimental Psychology: General, 131, 451-476.

Kirsch, I. J., Moore, T. J., Scoboria, A., \& Nicholls, S. S. (2002). The emperor's new drugs: An analysis of antidepressant medication data submitted to the U.S. Food and Drug Administration. Prevention \& Treatment, 5, 23a.

Klein, G. (1998). Sources of power: How people make decisions. Cambridge, MA: MIT Press.

Knaup, C., Koesters, M., Schoefer, D., Becker, T., \& Puschner, B. (2009). Effect of feedback of treatment outcome in specialist mental health care: Meta-analysis. British Journal of Psychiatry, 195, 15-22.

Koehler, D., \& Harvey, N. (2004). Blackwell handbook on decision making. Oxford: Blackwell Publishing.

Krynski, T. R., \& Tenenbaum, J. B. (2007). The role of causality in judgment under uncertainty. Journal of Experimental Psychology: General, 136(3), 430-450.

Kuyken, W. (2006). Evidence-based case formulation: Is the emperor clothed. In N. Tarrier (Ed.), Case formulation in cognitive behavior therapy (pp. 12-34). Hove: Routledge.

Kuyken, W., Fothergill, C. D., Musa, M., \& Chadwick, P. (2005). The reliability and quality of cognitive case formulation. Behaviour Research and Therapy, 43, 1187-1201.

Lewis, D. (2006). Causal decision theory. The Australasian Journal of Philosophy, 59, 5-30.

Litt, M. D., Kadden, R. M., \& Kabela-Cormier, E. (2009). Individualized assessment and treatment program for alcohol dependence: Results of an initial study to train coping skills. Addiction, 104, 1837-1848.

Lobban, F., Barrowclough, C., \& Jones, S. (2003). A review of the role of illness models in severe mental illness. Clinical Psychology Review, 23, 171-196.

Lombrozo, T. (2006). The structure and function of explanations. Trends in Cognitive Sciences, 10, 464-470.

Lombrozo, T., \& Vasilyeva, N. (2016). Causal explanation. In M. R. Waldmann (Ed.), Oxford handbook of causal reasoning. Oxford: Oxford University Press.

Maddux, W. E., \& Winstead, B. A. (2016). Psychopathology. Foundation for a contemporary understanding. Hove: Routledge.

Maher, P. (1987). Causality in the logic of decision. Theory and Decision, 22, 155-172.

Meder, B., Hagmayer, Y., \& Waldmann, M. R. (2008). Inferring interventional predictions from observational learning data. Psychonomic Bulletin \& Review, 15(1), 75-80.

Meder, B., Hagmayer, Y., \& Waldmann, M. R. (2009). The role of learning data in causal reasoning about observations and interventions. Memory \& Cognition, 37, 249-264.

Meder, B., Mayrhofer, R., \& Waldmann, M. R. (2014). Structure induction in diagnostic causal reasoning. Psychological Review, 121, 277-301.

Mijovic-Prelec, D., \& Prelec, D. (2010). Self-deception as self-signalling: A model and experimental evidence. Philosophical Transactions of the Royal Society B, 365, 227-240.

Mueller, S. M., Garcia-Retamero, R., Cokely, E., \& Maldonado, A. (2011). Causal beliefs and empirical evidence. Decision-making processes in two-alternative forced-choice tasks. Experimental Psychology, 58, 324-332.

Mueller, S. M., Garcia-Retamero, R., Galesic, M., \& Maldonado, A. (2013). The impact of domain-specific beliefs on decisions and causal judgments. Acta Psychologica, 144, $472-480$. 
Murdock, G. P. (1980). Theories of illness: A world survey. Pittsburgh, PA: University of Pittsburgh Press.

National Institute of Health. (2015). Bacterial infections. From https://medlineplus.gov/ healthtopics.html.

NICE. (2004). Clinical guideline 9: Eating disorders in over 8s: Management. From https://www.nice. org.uk/guidance/cg9/resources/eating-disorders-in-over-8s-management-27103918021.

NICE. (2005). Clinical guideline 26: Post-traumatic stress disorder: Management. From https:// www.nice.org.uk/guidance/cg26/resources/posttraumatic-stress-disorder-management975329451205.

NICE. (2009). Clinical guideline 90: Depression in adults: Recognition and management. From https://www.nice.org.uk/guidance/cg90/resources/depression-in-adults-recognitionand-management-975742636741.

Nozick, R. (1993). The nature of rationality. Princeton, NJ: Princeton University Press.

Osman, M. (2010). Controlling uncertainty: A review of human behavior in complex dynamic environments. Psychological Bulletin, 136, 65-86.

Patel, V. (1995). Explanatory models of mental illness in sub-Saharan Africa. Social Science and Medicine, 40, 1291-1298.

Pearl, J. (2000). Causality: Models, reasoning, and inference. Cambridge, MA: Cambridge University Press.

Pennington, N., \& Hastie, R. (1986). Evidence evaluation in complex decision making. Journal of Personality and Social Psychology, 51(2), 242-258.

Pennington, N., \& Hastie, R. (1988). Explanation-based decision making: Effects of memory structure on judgement. Journal of Experimental Psychology: Learning, Memory, and Cognition, 14(3), 521-533.

Pennington, N., \& Hastie, R. (1992). Explaining the evidence: Tests of the story model for juror decision making. Journal of Personality and Social Psychology, 62(2), 189-206.

Pennington, N., \& Hastie, R. (1993). Reasoning in explanation-based decision making. Cognition, 49, 123-163.

Persons, J. B. (2008). The case formulation approach to cognitive-behavior therapy. New York: Guildford Press.

Persons, J. B., \& Hong, J. J. (2016). Case formulation and the outcome of cognitive behavioral therapy. In N. Tarrier, \& J. Johnson (Eds.), Case formulation in cognitive behavior therapy (pp. 14-37). Howe: Routledge.

Quattrone, G., \& Tversky, A. (1984). Causal versus diagnostic contingencies: On selfdeception and on the voter's illusion. Journal of Personality and Social Psychology, 46, 237-248.

Resnik, M. D. (1987). Choices. An introduction to decision theory. Minneapolis: University of Minnesota Press.

Robinson, E., Sloman, S. A., Hagmayer, Y., \& Hertzog, C. (2010). Causality in solving economic problems. The Journal of Problem Solving, 3, 106-130.

Sackett, D. L., Rosenberg, W. M., Muir Gray, J. A., Haynes, R. B., \& Richardson, W. S. (1996). Evidence-based practice. What it is and what it isn't. British Medical Journal, 312, 71-72.

Savage, L. J. (1954). The foundations of statistics. New York: Wiley.

Schmidt, H. G., \& Rikers, R. M. (2007). How expertise develops in medicine: Knowledge encapsulation and illness script formation. Medical Education, 41, 1133-1139.

Schulte, D., Künzel, R., Pepping, G., \& Schulte-Bahrenberg, T. (1992). Tailor-made versus standardized therapy of phobic patients. Advances in Behaviour Research and Therapy, 14, 67-92.

Shimokawa, K., Lambert, M. J., \& Smart, D. W. (2010). Enhancing treatment outcome of patients at risk of treatment failure: Meta-analytic and mega-analytic review of 
psychotherapy quality assurance systems. Journal of Consulting and Clinical Psychology, 78, $298-311$.

Simon, D., Wills, C. E., \& Harter, M. (2009). Shared decision-making in mental health. In A. Edwards, \& G. Elwyn (Eds.), Shared decision-making in health care: Achieving evidence based patient choice (pp. 269-272). New York, NY: Oxford University Press.

Skyrms, B. (1982). Causal decision theory. The Journal of Philosophy, 79, 695-711.

Sloman, S. A. (2005). Causal models: How we think about the world and its alternatives. Oxford: Oxford University Press.

Sloman, S. A., Fernbach, P. M., \& Hagmayer, Y. (2010). Self-deception requires vagueness. Cognition, 115, 268-281.

Sloman, S. A., \& Hagmayer, Y. (2006). The causal psycho-logic of choice. Trends in Cognitive Sciences, 10, 407-412.

Sloman, S. A., \& Lagnado, D. A. (2005). Do we "do"? Cognitive Science, 29, 5-39.

Spirtes, P., Glymour, C., \& Scheines, R. (2000). Causation, prediction, and search. Cambridge: MIT Press.

Stephan, S., \& Waldmann, M. R. (2016). Answering causal queries about singular cases. In A. Papafragou, D. Grodner, D. Mirman, \& J. C. Trueswell (Eds.), Proceedings of the 38th Annual Conference of the Cognitive Science Society (pp. 2795-2801). Austin, TX: Cognitive Science Society.

Sterman, J. D. (2000). Business dynamics: Systems thinking and modeling for a complex world. Boston: McGraw-Hill.

Steyvers, M., Tenenbaum, J. B., Wagenmakers, E.-J., \& Blum, B. (2003). Inferring causal networks from observations and interventions. Cognitive Science, 27, 453-489.

Tarrier, N., \& Johnson, J. (2016). Case formulation in cognitive behavior therapy. Hove: Routledge.

Tenenbaum, J. B., Kemp, C., Griffiths, T. L., \& Goodman, N. D. (2011). How to grow a mind: Statistics, structure, and abstraction. Science, 331, 1279-1285.

Tenney, E. R., Cleary, M. D., \& Spellman, B. A. (2009). Unpacking the doubt in "beyond a reasonable doubt": Plausible alternative stories increase not guilty verdicts. Basic and Applied Social Psychology, 31, 1-8.

Von Neumann, J., \& Morgenstern, O. (1947). Theory of games and behavior. Princeton, NJ: Princeton University Press.

Vroom, V. H. (1964). Work and motivation. New York, NY: John Wiley and Sons.

Wald, A. (1945). Statistical decision functions which minimize the maximum risk. The Annals of Mathematics, 46(2), 265-280.

Waldmann, M. R. (1996). Knowledge-based causal induction. In D. R. Shanks, K. J. Holyoak, \& D. L. Medin (Eds.), Causal learning: Vol. 34. The psychology of learning and motivation (pp. 47-88). San Diego: Academic Press.

Waldmann, M. R. (2016). Oxford handbook of causal reasoning. Oxford: Oxford University Press.

Waldmann, M. R., Cheng, P. W., Hagmayer, Y., \& Blaisdell, A. P. (2008). Causal learning in rats and humans: A minimal rational model. In N. Chater, \& M. Oaksford (Eds.), The probabilistic mind. Prospects for Bayesian cognitive science (pp. 453-484). Oxford: University Press.

Waldmann, M. R., \& Hagmayer, Y. (2005). Seeing vs. doing: Two modes of accessing causal knowledge. Journal of Experimental Psychology: Learning, Memory, and Cognition, $31,216-227$.

Walsh, C. R., \& Sloman, S. A. (2011). The meaning of cause and prevent: The role of causal mechanism. Mind \& Language, 26, 21-52.

Wegwarth, O., Gaissmeier, W., \& Gigerenzer. (2009). Smart strategies for doctors and doctors-in-training: Heuristics in medicine. Medical Education, 43, 721-728. 
Weiner, B. (1985). An attributional theory of achievement motivation and emotion. Psychological Review, 92, 548-573.

Wessely, S., Bisson, J., \& Rose, S. (2000). A systematic review of brief psychological interventions ('debriefing') for the treatment of immediate trauma related symptoms and the prevention of post traumatic stress disorder. Cochrane Database of Systematic Reviews.

WHO. (1946). Preamble to the Constitution of the World Health Organization as adopted by the International Health Conference, New York, 19-22 June 1946 (Vol. 2, p. 100). New York: Official Records of the World Health Organization.

Winterbottom, A., Bekker, H. L., Conner, M., \& Montgomery, J. (2008). Does narrative information bias individual's decision making? A systematic review. Social Science and Medicine, 67, 2079-2088.

Witteman, C., \& Koele, P. (1999). Explaining treatment decisions. Psychotherapy Research, 9, $100-114$.

Yopchick, J. E., \& Kim, N. S. (2009). The influence of causal information on judgments of treatment efficacy. Memory \& Cognition, 37, 29-41.

Zsambok, C. E., \& Klein, G. (1997). Naturalistic decision making. Mahwah, NJ: Lawrence Erlbaum Associates. 